\title{
Estudio de caso de servicio de préstamo de libros electrónicos
}

\author{
Por Javier Clavero, Miquel Codina, Andrés Pérez y Marta Serrat-Brustenga
}

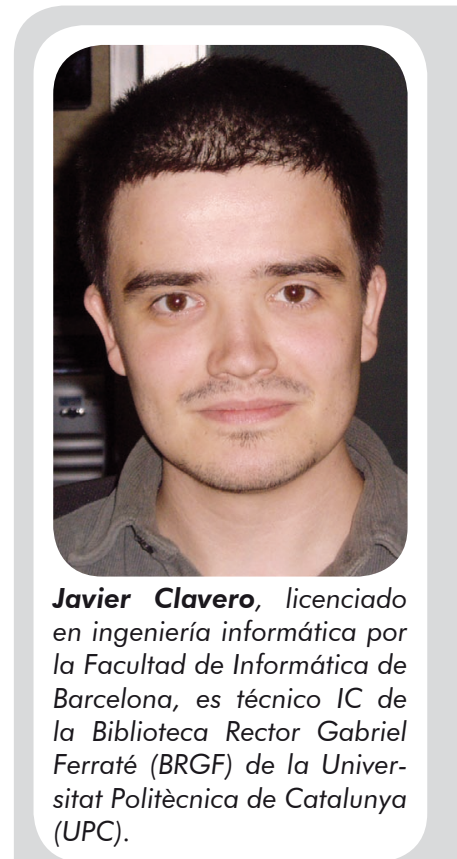

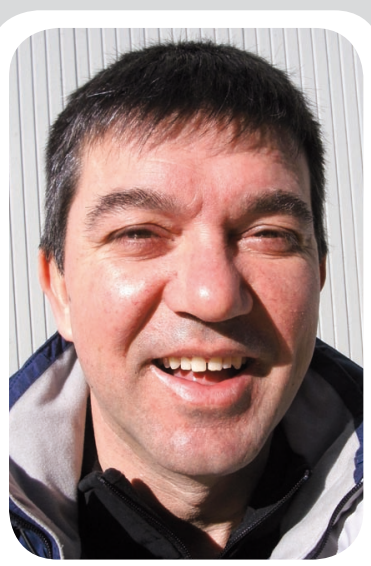

Miquel Codina, diplomado en biblioteconomía y documentación y licenciado en geografía e historia por la Univ. de Barcelona, es director de la Biblioteca Rector Gabriel Ferraté de la Univ. Politècnica de Catalunya (UPC).

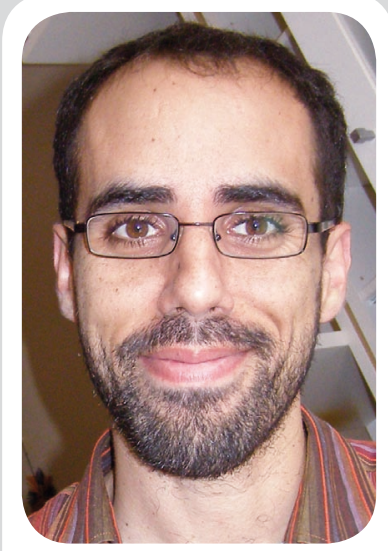

Andrés Pérez, diplomado en biblioteconomía y documentación por la Univ. de Barcelona y lic. en documentación por la Univ. Oberta de Catalunya, es responsable de la Unidad de Servicios Digitales de la BRGF de la UPC.

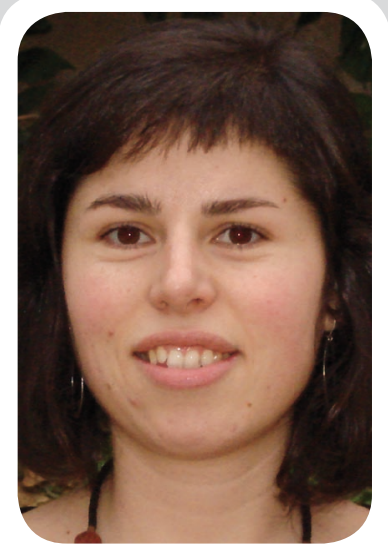

Marta Serrat-Brustenga, diplomada en biblioteconomía y documentación y licenciada en geografía por la Univ. de Barcelona, es bibliotecaria temática en la Biblioteca Rector Gabriel Ferraté de la UPC.

Resumen: La Biblioteca Rector Gabriel Ferraté (BRGF) de la Universidad Politécnica de Catalunya (UPC) ha puesto en marcha un servicio de préstamo de libros electrónicos. Se describe la experiencia con el proceso de evaluación de lectores de libros electrónicos existentes en el mercado, el contenido que se ha puesto a disposición de los usuarios a través de estos dispositivos y su funcionamiento, así como los resultados de dos meses de servicio. Finalmente, se apuntan las líneas de actuación más inmediata con los cuatro iRex iLiad 2nd Edition disponibles en la biblioteca.

Palabras clave: Libro electrónico, Biblioteca universitaria, iRex iLiad 2nd edition, Universidad Politécnica de Catalunya, Barcelona, España

\section{Title: A case study of lending service of electronic books}

Abstract: The Rector Gabriel Ferrate Library (BRGF) at the Technical University of Catalonia (UPC) has recently launched an electronic books lending service. This paper describes the experience of evaluating the e-book readers in the current market, the content to be made available on these devices, and how well they actually work, as well as the results of 2 months of ongoing service. Finally, it points to short-term future actions with all four copies of iRex iLiad 2 nd Edition available in the library.

Keywords: Electronic book, eBook, University library, Academic library, iRex iLiad 2nd edition, Polytechnical University of Catalonia, Barcelona, Spain

Clavero, Javier; Codina, Miquel; Pérez, Andrés; Serrat-Brustenga, Marta. "Servicio de préstamo de libros electrónicos de la Biblioteca Rector Gabriel Ferraté (BRGF)". El profesional de la información, 2009, marzo-abril, v. 18, n. 2, pp. 237-241.

DOI: $10.3145 /$ epi.2009.mar.15

LA BIBLIOTECA RECTOR GABRIEL FERRATÉ (BRGF) es la mayor del sistema bibliotecario de la Universitat Politécnica de Catalunya.

Ofrece servicio al Campus Norte de la $U P C$ en el que se ubican 3 cen- tros docentes: Facultat d'Informàtica de Barcelona (FIB), Escola Tècnica Superior d'Enginyeria de Telecomunicació de Barcelona (Etsetb) y Escola Tècnica Superior d'Enginyeria de Camins, Canals i Ports de Barcelona (Etseccpb) y 22 departamentos y centros de investigación, constituyendo uno de los polos estatales más importantes en investigación TIC, junto a la escuela de ingeniería civil mejor posicionada en España tanto en docencia como en investigación ${ }^{1}$. 
http://www.upc.edu

http://www.fib.upc.edu

http://www.etsetb.upc.edu

http://www-camins.upc.edu

En este contexto, la $B R G F$ sirve directamente a unos 8.000 usuarios, la mayoría de los cuales tiene conocimientos y requerimientos tecnológicos elevados y dispone desde hace tiempo (como el resto de la comunidad UPC) de servicios bibliotecarios y de colecciones digitales en plataformas tecnológicas:

- De producción externa integradas en Bibliotécnica, la biblioteca digital de la UPC donde desde hace algunos años se encuentran libros electrónicos.

\section{http://bibliotecnica.upc.edu/libres}

- De producción propia, recoCommons, y en la propia Bibliotécnica por lo que se refiere a los libros publicados por Ediciones UPC.

http://upcommons.upc.edu http://bibliotecnica.upc.edu/bib160/ gidas en el repositorio abierto $U P$ -

La $B R G F$ es consciente de que se encuentra en un entorno muy tecnológico y por ello apuesta por convertir la tecnología en una marca de la biblioteca. Uno de los aspectos a desarrollar es la biblioteca móvil o ubicua, desde la cual se podrá acceder a los servicios bibliotecarios permanentemente y desde cualquier lugar.

La $B R G F$ dispone de dos grupos dedicados al estudio e implementación de proyectos técnicos:

- BiT (Biblioteca y tecnología): Compuesto por 4 personas, tiene como tarea estudiar y evaluar innovaciones y novedades en hardware, software e internet. Propone ideas y acciones que permitan aumentar la cantidad y calidad de los servicios y productos de la $B R G F$ utilizando nuevas tecnologías.

- USD (Unidad de servicios digitales): Se encarga de los productos y servicios tecnológicos de la biblioteca, dando servicio no sólo al personal interno sino también a los usuarios, y ofreciendo apoyo en

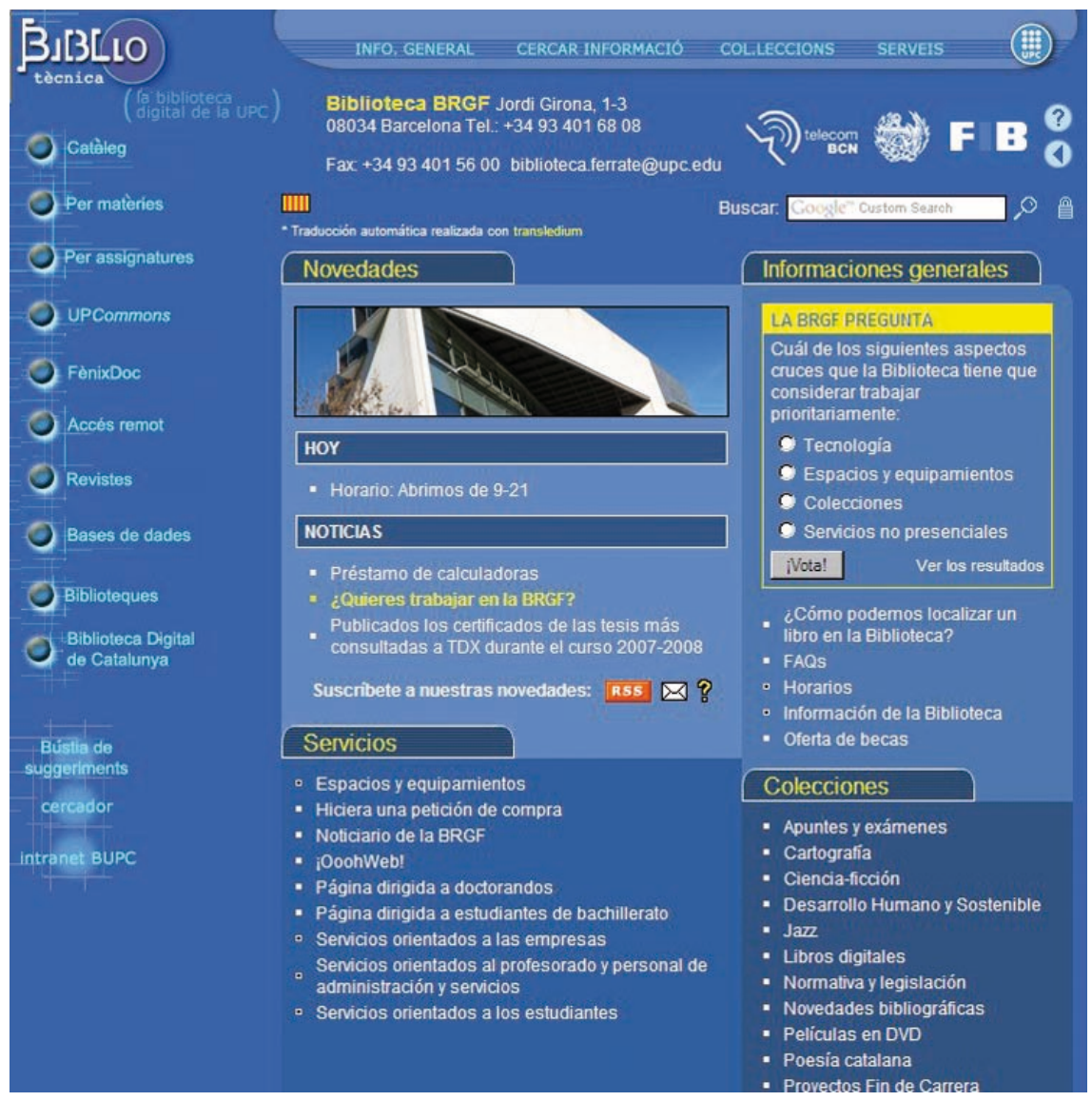

el uso de los dispositivos e infraestructuras digitales.

Gracias al trabajo conjunto de estos dos grupos, la $B R G F$ ha podido poner en marcha varios servicios, como son:

\section{- Avisos vía sms}

Envío de informaciones y avisos a los usuarios, como por ejemplo la disponibilidad de un portátil reservado.

\section{- OoohWeb!}

Portal donde se recopilan recursos web 2.0 que se consideran útiles para la actividad académica.

http://bibliotecnica.upc.edu/ bib160/serveis/ooohweb

\section{- CanalBIB}

Pantalla de televisión de grandes dimensiones donde se muestran informaciones de interés para la comunidad universitaria. Se han programado diversas aplicaciones que ofrecen información en tiempo real sobre la ocupación de aulas informáticas, la disponibilidad de recursos, etc.

\section{- Punto de información digital}

Terminal dedicado en exclusiva a suministrar información de la biblioteca partiendo de una nube de tags (etiquetas).

\section{Implementación del servicio}

Numerosos estudios y encuestas han venido apuntando durante los últimos tiempos a que:

- el libro electrónico es un sector emergente en el ámbito de la producción científica (y en la producción literaria en general);

- hay una demanda de libro electrónico (y de sus lectores portátiles) que se hace especialmente visible con el éxito de Kindle (el lector de Amazon) a finales de 2008 y principios de 2009;

- como mínimo, en el contexto de las universidades, el papel de las 
bibliotecas respecto al libro electrónico es muy relevante: los lectores manifiestan mayoritariamente conocer el nuevo formato a través de sus bibliotecas y también esperan que las bibliotecas les ofrezcan contenidos en este medio.

\section{"Se trabaja continuamente en nuevos servicios y proyectos tecnológicamente innovadores"}

Sensible a este contexto, la $B R G F$ decidió a principios de 2008 poner en marcha un servicio de préstamo de libros electrónicos. Al interés de la biblioteca se sumó el de la FIB, cuyo laboratorio de cálculo estaba buscando una solución para eliminar el papel en la redacción de las memorias de tesis de su nuevo máster en tecnologías de la información, lo cual significaba la necesidad de disponer de un sistema móvil de lectura para los integrantes de los tribunales de evaluación. De esta suma de intereses surgió la decisión de llevar a cabo un análisis conjunto de los lectores de libros electrónicos existentes en el mercado.

Primero se recopiló información, opiniones y características técnicas sobre los dispositivos lectores más relevantes, y seguidamente se hizo una comparativa para seleccionar los más adecuados para los fines perseguidos. Se eligieron 3:

- Sony Reader,

- Papyre, e

- iRex iLiad 2nd Edition.

Finalmente la $B R G F$ se decidió por el tercero debido a que, a pesar de ser el más caro, sus características técnicas eran superiores: mayor resolución de pantalla $(768 \times 1024$ píxeles, respecto a los 600x800 de sus competidores), procesador más rápido y, quizás lo más importante y el principal motivo de su elección, dispone de pantalla táctil que permite escribir notas en los documentos. Por otro lado, la FIB decidió adquirir un Papyre, para poder comparar en la práctica dispositivos diferentes.

\section{"Las principales fuentes son las colecciones de libros digitales presentes en Bibliotécnica y los depósitos de UPCommons"}

En septiembre de 2008 se utilizaron estos dos dispositivos en las defensas de los trabajos de fin de máster de la FIB. A los miembros del jurado se les facilitaron los aparatos lectores con las memorias de los estudiantes cargadas para que las pudieran leer el día de la defensa. El objetivo, como ya se ha comentado, era intentar eliminar la memoria en papel $\mathrm{y}$, al mismo tiempo, hacer más cómodo el proceso para los miembros del jurado evitándoles tener que cargar con las voluminosas memorias en papel de sus estudiantes. Los dispositivos fueron bien recibidos por la mayoría de los miembros de los jurados que los consideraron una buena alternativa al documento en papel, aunque es cierto que encontraron que estos equipos carecían de algunas funcionalidades que los harían aún más útiles como, por ejemplo, saltar a una página concreta dentro del documento. También hay que comentar que algún miembro de los jurados opinó que prefería el documento en papel o bien visualizar la memoria en su portátil.

Dada la buena aceptación, la $B R G F$ decidió poner en marcha el servicio de préstamo de libros electrónicos adquiriendo 3 lectores $i$ Rex iLiad más, o sea, 4 en total.

\section{"El índice de préstamos de este tipo de libros es muy superior al de cualquier libro en papel disponible en la BRGF"}

Se pasó a decidir qué contenidos deberían prestarse con el e-book y cómo se ofrecerían a los usuarios. Obviamente, lo primero que se consideró fue que tenía que estar relacionado con las disciplinas técnicas impartidas en la universidad y, más concretamente, con las de las tres escuelas presentes en el Campus Norte y a las que da servicio directamente la $B R G F$. Por ello se contemplaron como principales fuentes, por un lado, las colecciones de libros digitales presentes en Bibliotécnica, y por otro, los depósitos institucionales de e-prints, trabajos académicos y TDX (Tesis doctorales en red) que forman parte de UPCommons. En el caso de las primeras, dado que se trata de colecciones que pertenecen a editoriales externas a la universidad, se tuvo que estudiar el marco legal de las licencias de las que se disponía y averiguar si permitían el préstamo. Desgraciadamente la mayoría de las licencias no lo permitían lo que reducía enormemente el abanico de títulos. Para solventar este problema se decidió intentar llegar a acuerdos con las editoriales, para que concedieran su autorización para el uso de sus libros digitales en el contexto del nuevo servicio. Ediciones UPC, la editorial propia de la universidad, accedió sin problemas a que sus títulos formaran parte del nuevo servicio, pero todavía están en curso las conversaciones con otras editoriales para ver si es posible añadir sus tí- 
tulos. En el caso de UPCommons no fue necesario ya que todos sus títulos están disponibles en abierto en la Red y no hay ningún tipo de restricción que impida su uso en los lectores de libros electrónicos. Finalmente se decidió que también se añadiría una colección de novelas, adquiridas conjuntamente con los lectores, y se llegó a un acuerdo con la $F I B$ para destinar parte del presupuesto de adquisiciones para libros de informática en la compra de libros-e para su uso en los nuevos lectores.

\section{"Los mejores resultados se obtuvieron con ficheros pdf y mobi, un formato específico para lectores de libros electrónicos"}

A continuación se hicieron pruebas para ver si los formatos y tipos de fichero de cada colección eran aptos para los lectores iRex iLiad. Los mejores resultados se obtuvieron con los ficheros pdf, que todos conocemos, y mobi, un formato específico creado para lectores de libros electrónicos. Desgraciadamente se detectó un problema con los ficheros pdf creados con formato A4 y es que, a pesar de que se podían visualizar con el lector, al adaptarse al tamaño de pantalla la letra se reducía considerablemente y dificultaba la lectura. En la actualidad esto sigue siendo un problema pero se está trabajando en la adaptación de estos ficheros para que su visualización sea más agradable en los lectores. Con este fin se están evaluando programas que transforman los ficheros A4 a las medidas que se le especifiquen. De momento los resultados más prometedores se han conseguido con Mobipocket Creator y con Grammata Conversor, pero la conversión aún tiene algunos problemas. http://www.mobipocket.com http://www.grammata.es

Una vez realizado este estudio y a pesar de los problemas encontrados, la $B R G F$ decidió lanzar el servicio. La idea inicial era prestar por un lado un lector y por otro una tarjeta de memoria con los contenidos. Se tendrían diversas tarjetas con los distintos contenidos temáticos (tarjeta de libros de informática, tarjeta de novelas, etc.) y sería el usuario quien, en el momento del préstamo, seleccionaría la que más se adecuase a sus necesidades. Esta idea se descartó debido a que todos los contenidos podían introducirse en una única tarjeta, lo que llevó a pensar que sería más conveniente prestar lector y tarjeta conjuntamente con la tarjeta cargada con todos los contenidos posibles. De esta manera se simplificaba el proceso de préstamo y el usuario podía acceder a todas las colecciones independientemente de la tarjeta que tuviera. Es así como se imple-

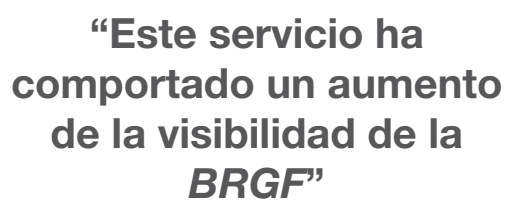

"Este servicio ha comportado un aumento BRGF" mentó el servicio y en la tarjeta de memoria de cada lector se introdujeron los títulos de 3 colecciones: Ediciones $U P C$, novelas, y libros especializados de informática de la editorial O'Reilly.

Se decidió que el préstamo se realizaría a través del catálogo de las bibliotecas de la $U P C$, al igual que se hace con el resto de los documentos en papel y para ello los 4 lectores fueron catalogados dentro del sistema. Siguiendo la misma normativa que para los libros en papel, cada lector se prestaría al usuario durante 10 días con la posibilidad de renovar hasta 4 veces.

El personal de la biblioteca entrega el lector con la batería cargada y con la tarjeta de memoria con los títulos de las colecciones disponibles, juntamente con una pequeña guía/manual de funcionamiento del dispositivo. Una vez devuelto, se comprueba si la batería necesita ser cargada y, como no se puede comprobar si el usuario ha modificado el contenido de la tarjeta de memoria, ésta siempre se formatea y se le vuelve a copiar el contenido original. De esta manera se garantiza que el siguiente usuario pueda gozar de los mismos contenidos.

El servicio se puso en funcionamiento a mediados de noviembre

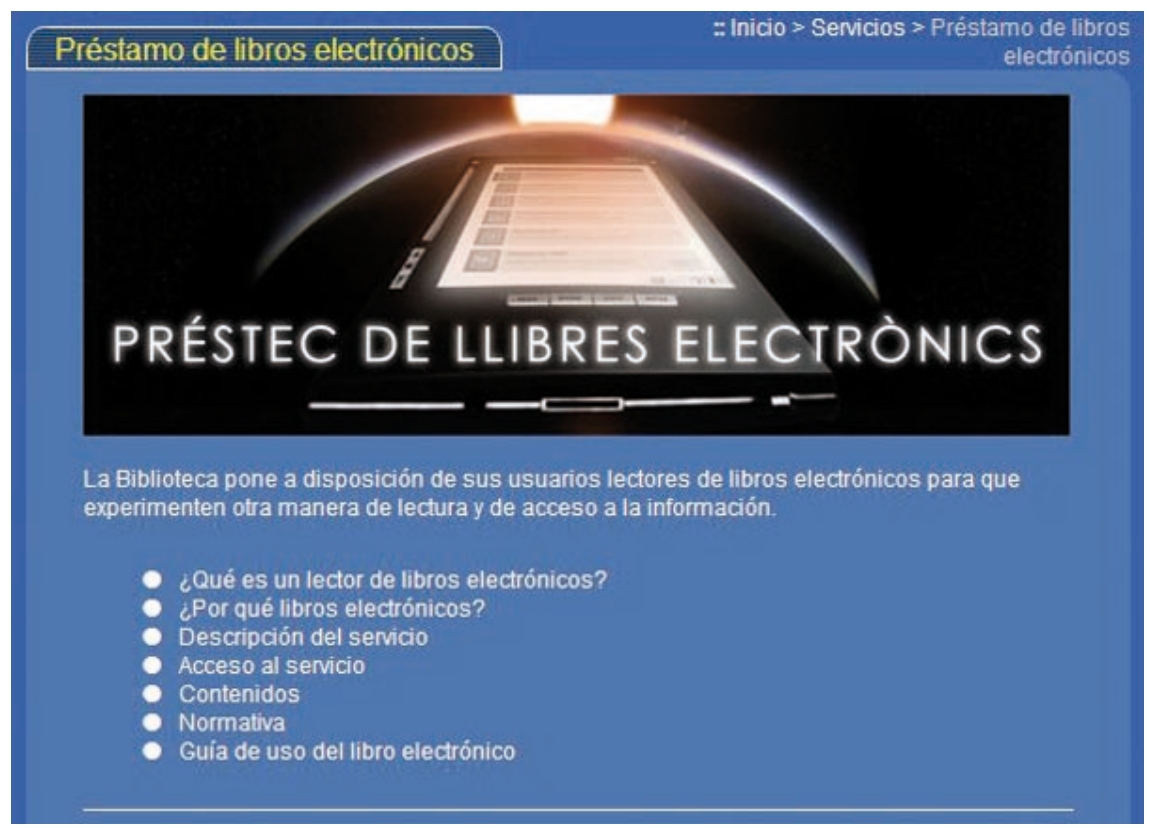


de 2008 y estará en fase de pruebas hasta febrero de 2009. Para impulsarlo se creó un apartado en la web de la biblioteca, donde se puede encontrar toda la información del funcionamiento y de los contenidos de los lectores, se imprimieron carteles promocionales que se repartieron por los diferentes mostradores de la biblioteca, se anunció por los canales electrónicos disponibles y fue recogido por el boletín de información de la universidad.

http://bibliotecnica.upc.edu/bib160/ serveis/ebooks/

\section{Resultados}

El lanzamiento del servicio ha comportado, a nivel cualitativo, un aumento de la visibilidad de la biblioteca en sus aspectos más tecnológicos. Profesores y estudiantes se han interesado por los lectores electrónicos y algunos los han utilizado no sólo para leer las colecciones de libros digitales sino también, por su novedad, para explorar su uso y funcionamiento técnico.

La difusión del servicio ha generado mensajes y reflexiones en el entorno profesional y en la blogosfera. Destacamos especialmente el comentario aparecido en el blog mantenido por Joaquín Rodríguez, Los futuros del libro:

http://weblogs.madrimasd.org/ futurosdellibro/archive/2008/12/03/ 108522.aspx
A nivel cuantitativo hay que subrayar que durante los 2 primeros meses de servicio, los cuatro lectores disponibles han sido ya prestados 15 veces, lo cual significa que han estado en préstamo más de un $90 \%$ del tiempo posible. El hecho de que los gadgets estén constantemente prestados hace que también tengan, cada uno de ellos, 3 reservas de promedio que es el máximo número permitido. Evidentemente basado en el factor "novedad", el índice de préstamos de este tipo de libros es muy superior al de cualquier libro en papel disponible en la $B R G F$.

\section{Conclusiones}

A pesar de que se trata de un sistema todavía deficitario tanto desde el punto de vista técnico como en contenidos del mercado editorial, el éxito del nuevo servicio entre nuestros usuarios (que creemos que son especialmente exigentes) nos ha llevado a decidir que continuaremos manteniendo, ampliando y actualizando el servicio de préstamo de libros electrónicos. En relación con aspectos críticos como el avance de la tecnología o la propiedad intelectual, para el futuro inmediato tendremos especialmente en cuenta:

- la ampliación de las colecciones accesibles con los lectores;

- la posibilidad de cargar libros en los lectores a petición del usuario, en lugar de prestar colecciones enteras;
- considerar el uso de los ebooks en actividades nuevas, como por ejemplo en talleres de habilidades informacionales.

El servicio de préstamo de libros electrónicos constituye un ejemplo a pequeña escala de las posibilidades de implementación de la tecnología en las bibliotecas, mejorando en calidad para ofrecer más y mejores servicios.

\section{Nota}

1. Según el ranking iberoamericano de Scimago la $U P C$ está en la posición número 1 en el área ingeniería civil y arquitectura:

http://investigacion.universia.net/isi/isi.html

En docencia, la UPC aparece asimismo destacada en el estudio del diario El mundo:

http://aula2.elmundo.es/aula/especiales/2007/ 50carreras/index.html

En investigación TIC (telecomunicaciones e informática) la $U P C$ también figura en los primeros puestos:

http://bibliotecnica.upc.edu/bib160/serveis/ bibliometria/Estudi_comparatiu_publicacio_ ETSETB_vs_Europa_97_07.pdf http://bibliotecnica.upc.es/observatori/

Javier Clavero, Miquel Codina, Andrés Pérez, Marta Serrat-Brustenga

Biblioteca Rector Gabriel Ferraté (BRGF), Universidad Politécnica de Catalunya (UPC)

Jordi Girona 1-3, 08034 Barcelona, España.

javier.clavero@upc.edu

miquel.codina@upc.edu

andres.perez@upc.edu

marta.serrat@upc.edu

\section{Suscripción EPI sólo online}

Pensando sobre todo en los posibles suscriptores latinoamericanos, ya no es obligatorio pagar la suscripción impresa de EPI para acceder a la online.

EPI se ofrece a instituciones en suscripción "sólo online"

a un precio considerablemente más reducido (90 euros/año), puesto que en esta modalidad no hay que cubrir los gastos de imprenta ni de correo postal. 


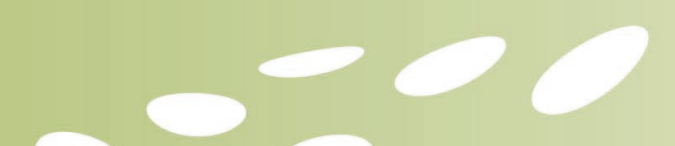

$\because 00$ and and

\section{Colección Médica Española de Elsevier Doyma}

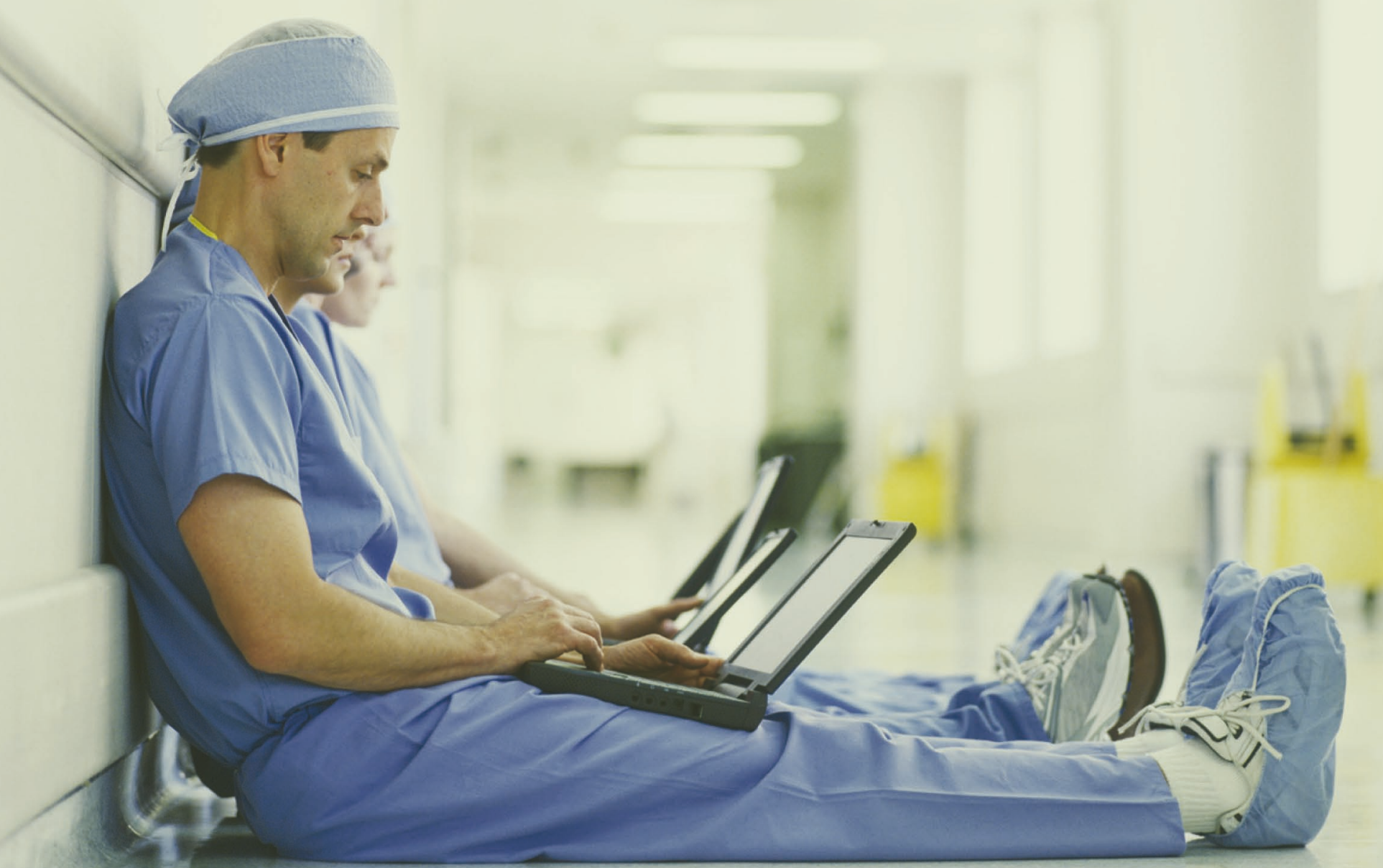

Cincuenta revistas médicas de alta calidad en español serán añadidas a ScienceDirect incluyendo títulos importantes como:

- Archivos de Bronconeumología

- Medicina Clínica

- Revista Española de Cardiología
- Formación Médica Continuada

- Medicine

- Anales de Pediatría Continuada

La colección incluye:

- Revistas de Investigación clínica con artículos originales, revisiones y casos clínicos, la mayoría de los cuales son publicaciones oficiales de las principales sociedades médicas españolas.

- Revistas de Formación Médica Continuada (FMC), un gran número de ellas están respaldadas por sociedades de prestigio reconocido.

Periodo de prueba disponible para las instituciones tryit@elsevier.com 